\title{
Correlated Electron and Nuclear Dynamics in Strong Field Photoionization of $\mathbf{H}_{2}^{+}$
}

\author{
R. E. F. Silva, ${ }^{1}$ F. Catoire, ${ }^{2}$ P. Rivière, ${ }^{1}$ H. Bachau, ${ }^{2}$ and F. Martín ${ }^{1,3}$ \\ ${ }^{1}$ Departamento de Química, Módulo 13, Universidad Autónoma de Madrid, 28049 Madrid, Spain \\ ${ }^{2}$ Centre des Lasers Intenses et Applications CNRS-CEA-Université Bordeaux I, 351 Cours de la Libération, Talence F-33405, France \\ ${ }^{3}$ Instituto Madrileño de Estudios Avanzados en Nanociencia, Cantoblanco, 28049 Madrid, Spain
}

(Received 17 October 2012; published 12 March 2013)

\begin{abstract}
We present a theoretical study of $\mathrm{H}_{2}^{+}$ionization under strong IR femtosecond pulses by using a method designed to extract correlated (2D) photoelectron and proton kinetic energy spectra. The results show two distinct ionization mechanisms - tunnel and multiphoton ionization-in which electrons and nuclei do not share the energy from the field in the same way. Electrons produced in multiphoton ionization share part of their energy with the nuclei, an effect that shows up in the 2D spectra in the form of energy-conservation fringes similar to those observed in weak-field ionization of diatomic molecules. In contrast, tunneling electrons lead to fringes whose position does not depend on the proton kinetic energy. At high intensity, the two processes coexist and the 2D plots show a very rich behavior, suggesting that the correlation between electron and nuclear dynamics in strong field ionization is more complex than one would have anticipated.
\end{abstract}

DOI: 10.1103/PhysRevLett.110.113001

PACS numbers: $33.20 . \mathrm{Xx}, 33.60 .+\mathrm{q}, 33.80 . \mathrm{Rv}$

The interaction of atoms and molecules with intense infrared laser pulses has been the object of continuous research for more than two decades [1-9]. Since the potential induced by such lasers on the electrons is comparable to or even stronger than that generated by the nuclei, the resulting electron dynamics is significantly different from that of the isolated system, which makes these lasers ideal tools to achieve electronic control [10-13]. Strong fields can efficiently excite and ionize atoms and molecules. The electrons, which can be ejected following either multiphoton absorption or tunneling, can either directly reach the detector after having been repeatedly accelerated and decelerated by the field [direct electrons (DE)] or recollide with the ionic core within an optical cycle [rescattered electrons (RE)] [14,15]. Only a small fraction of the ejected electrons rescatter, but this fraction is responsible for important nonlinear phenomena such as high-harmonic generation (HHG). In this process, high-energy photons are emitted as a result of electron recombination with the ionic core. HHG is currently used to produce ultrashort extreme ultraviolet laser pulses and trains of these pulses [16-19], and also to uncover multielectron dynamics in atoms and molecules [13,20] or the structure of atomic and molecular orbitals in the so-called orbital tomography [10,21,22].

Rescattered electrons that do not recombine with the ion also leave their signature in the photoelectron spectra at relatively high energies, typically between $2 U_{p}$ and $10 U_{p}$ $[23,24]$, where $U_{p}=I / 4 \omega^{2}$ is the electron ponderomotive energy (in a.u.), $I$ is the laser intensity, and $\omega$ its frequency. Because of their high energy, in contrast with that of direct electrons which is $\leq 2 U_{p}$, RE can be used as signal and $\mathrm{DE}$ as reference to image atomic and molecular structure by photoelectron holography $[20,25]$.
Compared to atoms, the study of strong-field electron dynamics in molecules, in particular ionization, offers a richer perspective due to the additional degrees of freedom associated with the nuclei. Indeed, several phenomena due to the multicenter character of the molecular potential have already been discovered, such as charge-resonanceenhanced ionization [26] and light-induced-electron diffraction [27-29]. The induced electron dynamics can also be used to learn about the nuclear motion in molecules, e.g., vibration and dissociation. A few attempts in this direction have been reported in the context of HHG [30] and light-induced-electron diffraction [12], where atomic displacements have been detected by measuring the harmonic emission and the ionized electrons, respectively. For light molecules with relatively fast nuclear dynamics, the other way around is also possible: one can learn about electron dynamics by looking at the atomic or molecular ions produced in the ionization of the parent molecule. This has been achieved in strong field ionization of $\mathrm{H}_{2}$ [31], but also in weak-field few-photon ionization of $\mathrm{H}_{2}$ by ultrashort UV and extreme ultraviolet pulses [32].

Early work has shown that electron and nuclear dynamics may not always be disentangled [33]. This is so when, e.g., ionized electrons are slow and the Born-Oppenheimer (BO) approximation is no longer valid. In this case, the electron and nuclear dynamics must be considered on an equal footing. So, ideal experiments are those in which electrons and ions are detected in coincidence [34], since this provides information about how the energy is shared between them. The correlated electron and nuclear dynamics can be visualized by plotting the ionization yield as a function of both the electron and ion kinetic energies [35-38] (2D plots). State-of-the-art multicoincidence detection techniques are now able to provide such 
information. They have been successfully used to investigate the correlated electron and nuclear dynamics in dissociative ionization of $\mathrm{H}_{2}$ by synchrotron radiation $[39,40]$. So the natural question is if similar coincidence techniques can shed light about the energy sharing mechanisms in the presence of a strong field. This is relevant because, at high field intensities, nonadiabatic effects are expected to appear, such as those due to the appearance of avoided crossings between dressed molecular states in the presence of the laser field, that may lead to changes in the rovibrational spectra of diatomic molecules [41] and can be used to control molecular photodissociation [42].

In this Letter we address the above question by using a newly developed theoretical method that allows us to extract correlated photoelectron and nuclear-kinetic energy spectra of $\mathrm{H}_{2}^{+}$molecules under intense ultrashort laser pulses. This method is an extension of the resolvent technique originally proposed for atoms $[43,44]$. The calculated 2D plots suggest that electrons resulting from multiphoton ionization and tunneling ionization do not share energy with the nuclei in the same way. In particular, tunneling electrons, either ejected directly or after rescattering, are reluctant to share their energy with the nuclei, while electrons produced in multiphoton ionization actually share part of their energy. When these processes coexist, the calculated 2D plots exhibit a very rich behavior, suggesting that the correlation between electron and nuclear dynamics in strong field ionization is more complex than anticipated, and that, in general, the nuclear motion cannot be ignored in realistic simulations of strong field ionization in molecules.

The time-dependent Schrödinger equation (TDSE) is solved numerically in a two-dimensional grid for $z$ and $R$, where $z$ is the electronic coordinate parallel to the linear polarization axis of the field, and $R$ is the internuclear distance. In atomic units (a.u.), the TDSE reads

$i \frac{d \Psi(z, R, t)}{d t}=\left[\hat{H}_{e l}(z, R)+\hat{T}(R)+\hat{V}(z, t)\right] \Psi(z, R, t)$,

where $\hat{H}_{e l}=\hat{T}_{e l}+\hat{V}_{e N}+1 / R$ is the electronic Hamiltonian of $\mathrm{H}_{2}^{+}$, which includes the electronic kinetic energy and the electron-nuclear potential, $\hat{T}(R)$ is the nuclear-kinetic energy (NKE), and $\hat{V}=\hat{z} \hat{E}(t) \quad(\hat{V}=$ $\hat{p} \hat{A}(t))$ is the laser potential in length (velocity) gauge. A standard soft-core potential is used to treat the Coulomb singularity [45]. We have used a box with $|z|<3000$ a.u. and $R<30$ a.u., with uniform grid spacings of $\Delta z=$ 0.1 a.u. and $\Delta R=0.05$ a.u. The propagation was performed by using the Crank-Nicolson split-operator method with $\Delta t=0.02$ a.u. We assume that the pulse has a $\sin ^{2}$ envelope.

To extract the physical observables, we have extended the method of Ref. [44] to account for both the electron and the nuclei. We define the electronic and nuclear resolvent operators (RO)

$$
\begin{gathered}
R_{\epsilon_{e}}^{n}\left(E_{e}\right)=\frac{\epsilon_{e}{ }^{n}}{\left[\hat{H}_{e}-1 / R-E_{e}\right]^{n}-i \epsilon_{e}{ }^{n}}, \\
R_{\epsilon_{\mu}}^{n}\left(E_{\mu}\right)=\frac{\epsilon_{\mu}{ }^{n}}{\left[\hat{T}(R)+1 / R-E_{\mu}\right]^{n}-i \epsilon_{\mu}{ }^{n}},
\end{gathered}
$$

where $E_{e}$ is the asymptotic electron kinetic energy with resolution $\epsilon_{e}, E_{\mu}$ is the asymptotic nuclear kinetic energy with resolution $\epsilon_{\mu}$, and $n$ is the order of the RO. We have used $n=2, \epsilon_{e}=0.004$ a.u., and $\epsilon_{\mu}=0.02$ a.u.

We now define a new function $\Psi^{\prime}$ that results from applying the above operators to the solution of the TDSE at $t=t_{\max }$ (the largest value of time in the propagation)

$$
\left|\Psi^{\prime}\right\rangle \equiv R_{\epsilon_{\mu}}^{n}\left(E_{\mu}\right) R_{\epsilon_{e}}^{n}\left(E_{e}\right)\left|\Psi\left(t=t_{\max }\right)\right\rangle .
$$

The differential probability density of finding the system with fragment energies $E_{e}$ and $E_{\mu}$ is thus given by

$$
\rho\left(E_{e}, E_{\mu}\right)=\frac{P\left(E_{e}, E_{\mu}\right)}{\alpha_{e} \alpha_{\mu}},
$$

where

$$
P\left(E_{e}, E_{\mu}\right)=\left\langle\Psi^{\prime} \mid \Psi^{\prime}\right\rangle
$$

and $\alpha_{i}=\epsilon_{i} \frac{\pi}{n} \csc \left(\frac{\pi}{2 n}\right)$, with $i=(e, \mu)$. At $t=\infty$, the total energy $E$ of the system is just $E_{e}+E_{\mu}$. Thus, for large enough $t$ (cf. $z$ and $R$ ), the first RO, $R_{\epsilon_{e}}^{n}\left(E_{e}\right)$, selects a value of the asymptotic electronic energy in the electronic continuum and the second one, $R_{\epsilon_{\mu}}^{n}\left(E_{\mu}\right)$, a value of the asymptotic nuclear energy in the dissociative continuum. The modulus square of the resulting wave function $P\left(E_{e}, E_{\mu}\right)$ is thus proportional to the probability density of finding an electron and the two protons with energy $E_{e}$ and $E_{\mu}$, respectively. We notice that, since in practice integration of the TDSE must be stopped at a finite time $t=t_{\max }$, we are in fact making use of the $\mathrm{BO}$ approximation in the projection given in Eq. (4) (but not in the time propagation). However, we have checked that the results remain practically invariant as $t_{\max }$ is increased, which means that non-BO effects are correctly included. Another indication of the latter is that doubly differential probabilities obtained in length and velocity gauges are very similar, with a relative error smaller than $1 \%$ at the higher electron energies and smaller than $10 \%$ close to the ionization threshold. All results shown below were obtained in the length gauge, unless otherwise stated.

Figure 1 shows the correlated photoelectron-nuclear kinetic energy spectra resulting from four different pulses with wavelength $(\lambda)$, duration $(T)$, and intensity (I): (a) $\lambda=400 \mathrm{~nm}, T=16 \mathrm{fs}$, and $I=10^{14} \mathrm{~W} / \mathrm{cm}^{2}$, (b) $\lambda=400 \mathrm{~nm}, T=16 \mathrm{fs}$, and $I=4 \times 10^{14} \mathrm{~W} / \mathrm{cm}^{2}$, (c) $\lambda=800 \mathrm{~nm}, T=32 \mathrm{fs}$, and $I=10^{14} \mathrm{~W} / \mathrm{cm}^{2}$, and (d) $\lambda=800 \mathrm{~nm}, T=16 \mathrm{fs}$, and $I=2 \times 10^{14} \mathrm{~W} / \mathrm{cm}^{2}$. They correspond, respectively, to values of the Keldysh parameter $\gamma=\sqrt{I_{p} / 2 U_{p}}=3.2,1.6,1.6$, and $1.1\left(I_{p}\right.$ is 

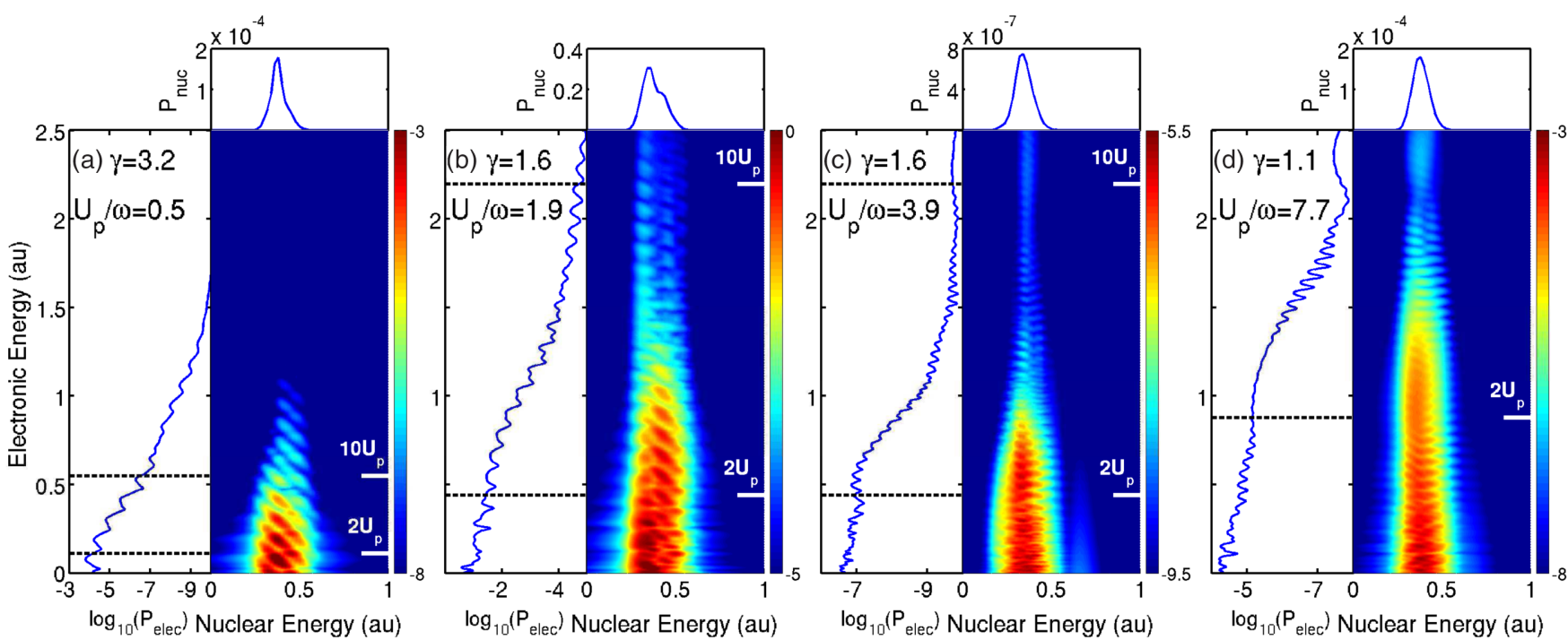

FIG. 1 (color online). Density plots for the correlated photoelectron and nuclear-kinetic energy spectra resulting from $\mathrm{H}_{2}^{+}$photoionization by using the following pulses: (a) $\lambda=400 \mathrm{~nm}, T=16 \mathrm{fs}$, and $I=10^{14} \mathrm{~W} / \mathrm{cm}^{2}$, (b) $\lambda=400 \mathrm{~nm}, T=16 \mathrm{fs}$, and $I=4 \times 10^{14} \mathrm{~W} / \mathrm{cm}^{2}$, (c) $\lambda=800 \mathrm{~nm}, T=32 \mathrm{fs}$, and $I=10^{14} \mathrm{~W} / \mathrm{cm}^{2}$, and (d) $\lambda=800 \mathrm{~nm}, T=16 \mathrm{fs}$, and $I=2 \times 10^{14} \mathrm{~W} / \mathrm{cm}^{2}$. The corresponding projections (singly differential probabilities) in electronic energy $\left(P_{\text {elec }}\right)$ and nuclear energy $\left(P_{\text {nuc }}\right)$ are shown on the left and on top of each panel. All panels include the values of the Keldysh parameter $\gamma$, the ratio between the ponderomotive energy and the photon energy $U_{p} / \omega$, and two and ten times $U_{p}$.

the vertical ionization energy), which cover different ionization regimes, from the multiphoton regime [Fig. 1(a)] to the tunneling regime [Fig. 1(d)]. In Fig. 1(a) one can see energy conservation lines satisfying the formula $N \omega \simeq$ $E_{e}+E_{\mu}+D_{2 H^{+}}$, where $D_{2 H^{+}}$is the energy required to produce two protons at infinite internuclear distance, and $N$ indicates the number of absorbed photons. The appearance of these lines indicates that the excess photon energy is shared between the ejected electron and protons. This energy sharing is only efficient within the Franck-Condon region, i.e., in the interval of nuclear-kinetic energies $0.25-0.55$ a.u. This is the usual behavior observed in weak-field ionization of diatomic molecules [35-37,39], and also, as pointed out very recently, in multiphoton above threshold ionization of $\mathrm{H}_{2}^{+}$[38]. As one increases the intensity without changing the photon energy [Fig. 1(b)], the calculated 2D spectrum exhibits a more complex structure. In this case, $\gamma$ is closer to 1 , so the tunneling process is expected to occur. In addition to the diagonal lines observed in the previous case, one can observe horizontal lines approximately separated by $\omega$ indicating that the energy taken by the electrons from the field is not shared with the nuclei. Horizontal lines are the dominant pattern for NKE between 0.2 and 0.3 a.u., and they are visible up to photoelectron energies close to $10 U_{p}$. For NKE around 0.4 a.u., there is no clear dominant pattern. The spectrum shown in Fig. 1(c) for the same value of $\gamma$ but obtained with an IR pulse that is four times less intense and contains photons with half the energy is dominated by horizontal lines. The same occurs if one considers even higher intensities [Fig. 1(d)]. In the latter case, as the Keldysh parameter is close to 1 and $U_{p}$ is about eight times the photon energy at maximum intensity, the ionization mechanism is clearly dominated by nonperturbative effects.

To understand how the different structures build up in the 2D spectra, we have evaluated in the velocity gauge the ionization probabilities at the zeros of the vector potential $A(t)$ for the case $\lambda=400 \mathrm{~nm}, T=16 \mathrm{fs}$, and $I=$ $4 \times 10^{14} \mathrm{~W} / \mathrm{cm}^{2}$ [Fig. 1(b)]. In this gauge, the lasermolecule interaction vanishes at the zeros of $A$, and consequently, the Hamiltonian is identical to that of a free molecule. Also, the kinematic momentum of the electron coincides with its canonical momentum, which is convenient to compare 2D spectra obtained at different times with that obtained at the end of the simulation. Figure 2(a) shows the 2D spectrum after the first few cycles. During this time interval, the intensity does not reach a large enough value to induce tunneling, and consequently, the resulting spectra is very similar to that obtained in the pure multiphoton regime. When the peak intensity is reached, one can see the appearance of horizontal fringes below $2 U_{p}$ in the region of NKE $\sim 0.25$ a.u. [see Fig. 2(b)]. This is the signature of tunneling electrons directly escaping from the molecule (DE). A cycle later, the 2D spectrum shows the appearance of horizontal fringes up to $\sim 10 U_{p}$ [see Fig. 2(c)], which is the signature of tunneling electrons driven back by the field and subsequently rescattered (RE). According to the threestep model [15], these electrons are expected to appear $\sim 0.65(2 \pi / \omega)$ after the DE. The subsequent evolution of the system repeatedly generates similar patterns, which interfere with each other and thus lead to the complex 2D spectrum of Fig. 1(b). That the origin of the horizontal fringes is 


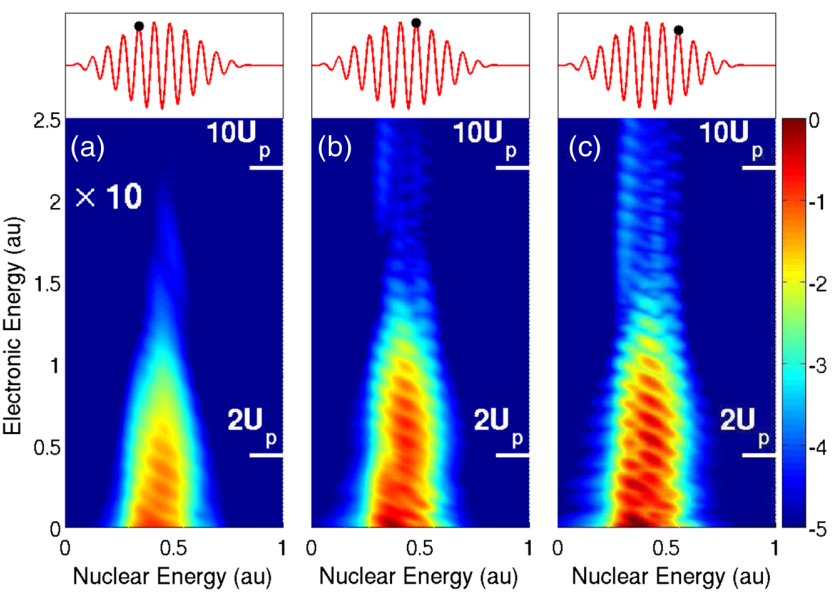

FIG. 2 (color online). Time evolution of the density plots for the correlated photoelectron and nuclear-kinetic energy spectra resulting from $\mathrm{H}_{2}^{+}$photoionization by using the a pulse with $\lambda=400 \mathrm{~nm}, T=16 \mathrm{fs}$, and $I=4 \times 10^{14} \mathrm{~W} / \mathrm{cm}^{2}$. The time values are indicated by black dots on the electric field displayed on top of each panel.

tunneling ionization is confirmed by calculations performed on a one-dimensional $\mathrm{He}^{+}$system represented by a soft Coulomb potential that provides the same ionization energy as for $\mathrm{H}_{2}^{+}$. The resulting $\mathrm{He}^{+}$electron kinetic energy spectra resemble those of $\mathrm{H}_{2}^{+}$for electron energies well above $2 U_{p}$, i.e., in the region where ionization comes almost exclusively from tunneling electrons. At low electron kinetic energies, interferences between multiphoton and tunneling ionization leads to complex patterns that are different in $\mathrm{He}^{+}$and $\mathrm{H}_{2}^{+}$ due to the molecular character of multiphoton ionization.

An interesting feature of the 2D spectrum shown in Fig. 1(b) is that the NKE distribution exhibits some structure and is significantly wider than that expected from the FranckCondon principle (which is close to the NKE distributions observed in the other three cases). This can be explained with the help of Fig. 3, which shows the variation with time of ionization and vibrational-excitation probabilities, as well as that of the average value of the internuclear distance, for the $400 \mathrm{~nm}, 4 \times 10^{14} \mathrm{~W} / \mathrm{cm}^{2}$, and $800 \mathrm{~nm}, 10^{14} \mathrm{~W} / \mathrm{cm}^{2}$ cases [Figs. 1(b) and 1(c)]. As can be seen, for $400 \mathrm{~nm}$, there is a large degree of vibrational excitation, which is the consequence of Rabi oscillations between the $1 s \sigma_{g}$ and $2 p \sigma_{u}$ states following a stepladder mechanism similar to that previously described in $\mathrm{H}_{2}[46,47]$. The coherent population of the different vibrational states launches a nuclear wave packet in the $1 s \sigma_{g}$ state that moves considerably during the pulse duration. As a consequence of this, the average internuclear distance increases from $\sim 1.9$ a.u. up to $\sim 2.7$ a.u. at the end of the pulse. Thus, when ionization occurs in the second half of the pulse, the available NKE is smaller because the internuclear distance is larger. This leads to the broadening of the NKE distribution when tunneling electrons appear. For $800 \mathrm{~nm}$ (and all the other cases), the effect is negligible and the NKE distribution follows a typical Franck-Condon behavior.
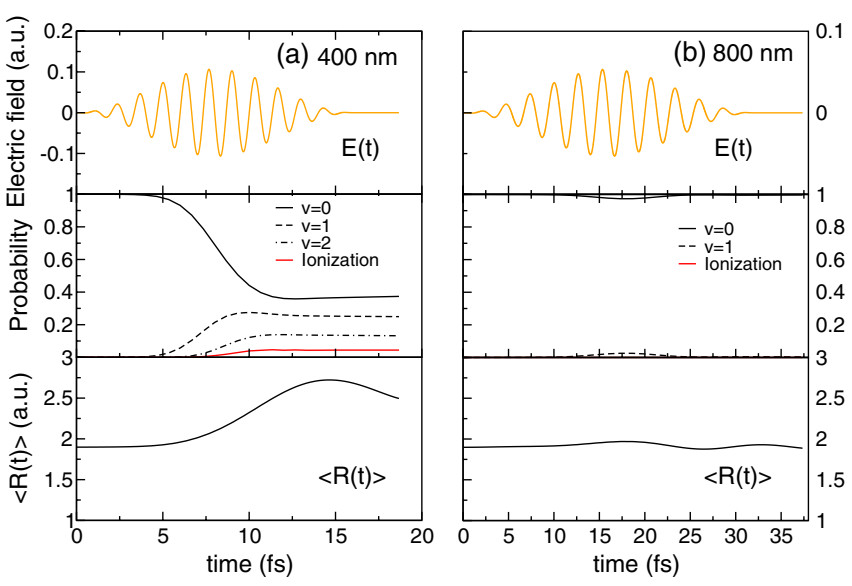

FIG. 3 (color online). Time evolution of different observables for the $\left(400 \mathrm{~nm}, 4 \times 10^{14} \mathrm{~W} / \mathrm{cm}^{2}\right)$ (a) and $\left(800 \mathrm{~nm}, 10^{14} \mathrm{~W} / \mathrm{cm}^{2}\right)$ (b) pulses. Electric field (top), population of the lowest vibrational states and ionization probability (middle figure), and mean value of the internuclear distance $\langle R(t)\rangle$ (bottom).

In conclusion, we have presented a new method to obtain correlated photoelectron and nuclear-kinetic energy spectra in strong field ionization of $\mathrm{H}_{2}^{+}$. The results show that electrons produced in multiphoton ionization share their energy with the nuclei, an effect that shows up in the 2D spectra in the form of energy-conservation fringes similar to those observed in weak-field ionization of diatomic molecules. In contrast, electrons resulting from tunneling ionization lead to fringes whose position does not depend on the proton kinetic energy; i.e., the molecular character is somewhat lost. At high intensity, the two processes coexist and the 2D plots exhibit a complex structure, thus showing that the correlation between electron and nuclear dynamics in strong field ionization is involved as a result of the interplay between the electronic and the nuclear motion.

This work was accomplished with an allocation of computer time from Mare Nostrum BSC and CCC-UAM, and was partially supported by the MICINN Projects No. FIS2010-15127, No. ACI2008-0777, and No. CSD 2007-00010, the ERA-Chemistry Project No. PIM2010EEC-00751, the European Grants No. MCITN CORINF and No. MC-RG ATTOTREND, the European COST Action CM0702, and the Advanced Grant of the European Research Council, Grant No. XCHEM 290853. R.E. F. S. acknowledges a Ph.D. contract from ITN CORINF. P. R. acknowledges a Juan de la Cierva contract grant from MICINN.

[1] P. B. Corkum, N. H. Burnett, and M. Y. Ivanov, Opt. Lett. 19, 1870 (1994).

[2] T. Seideman, M. Y. Ivanov, and P. B. Corkum, Phys. Rev. Lett. 75, 2819 (1995). 
[3] J. H. Posthumus, Rep. Prog. Phys. 67, 623 (2004).

[4] T. Ergler, A. Rudenko, B. Feuerstein, K. Zrost, C. D. Schröter, R. Moshammer, and J. Ullrich, Phys. Rev. Lett. 97, 193001 (2006).

[5] C. I. Blaga, F. Catoire, P. Colosimo, G. G. Paulus, H. G. Muller, P. Agostini, and L. F. DiMauro, Nat. Phys. 5, 335 (2009).

[6] H. Akagi, T. Otobe, A. Staudte, A. Shiner, F. Turner, R. Dörner, D. M. Villeneuve, and P. B. Corkum, Science 325, 1364 (2009).

[7] M. Odenweller, N. Takemoto, A. Vredenborg, K. Cole, K. Pahl, J. Titze, L. P. H. Schmidt, T. Jahnke, R. Dörner, and A. Becker, Phys. Rev. Lett. 107, 143004 (2011).

[8] I. A. Bocharova, R. Karimi, E. Penka, J.-P. Brichta, P. Lassonde, X. Fu, J.-C. Kieffer, A.D. Bandrauk, I. Litvinyuk, J. Sanderson et al., Phys. Rev. Lett. 107, 063201 (2011).

[9] A. E. Boguslavskiy, J. Mikosch, A. Gijsbertsen, M. Spanner, S. Patchkovskii, N. Gador, M. J. J. Vrakking, and A. Stolow, Science 335, 1336 (2012).

[10] J. Itatani, J. Levesque, D. Zeidler, H. Niikura, H. Pépin, J.-C. Kieffer, P. B. Corkum, and D. M. Villeneuve, Nature (London) 432, 867 (2004).

[11] M. Uiberacker, T. Uphues, M. Schultze, A. J. Verhoef, V. Yakovlev, M.F. Kling, J. Rauschenberger, N. M. Kabachnik, H. Schröder, M. Lezius et al., Nature (London) 446, 627 (2007).

[12] C. I. Blaga, J. Xu, A. D. DiChiara, E. Sistrunk, K. Zhang, P. Agostini, T. A. Miller, L. F. DiMauro, and C. D. Lin, Nature (London) 483, 194 (2012).

[13] O. Smirnova, Y. Mairesse, S. Patchkovskii, N. Dudovich, D. M. Villeneuve, P. B. Corkum, and M. Y. Ivanov, Nature (London) 460, 972 (2009).

[14] J. L. Krause, K. J. Schafer, and K. C. Kulander, Phys. Rev. Lett. 68, 3535 (1992).

[15] P. B. Corkum, Phys. Rev. Lett. 71, 1994 (1993).

[16] P. M. Paul, E. S. Toma, P. Breger, G. Mullot, F. Auge, P. Balcou, H. G. Muller, and P. Agostini, Science 292, 1689 (2001).

[17] M. Hentschel, R. Kienberger, C. Spielmann, G. A. Reider, N. Milosevic, T. Brabec, P. B. Corkum, U. Heinzmann, M. Drescher, and F. Krausz, Nature (London) 414, 509 (2001).

[18] T. Remetter, P. Johnsson, J. Mauritsson, K. Varjú, Y. Ni, F. Lépine, E. Gustafsson, M.F. Kling, J. I. Khan, R. B. López-Martens et al., Nat. Phys. 2, 323 (2006).

[19] F. Krausz and M. Y. Ivanov, Rev. Mod. Phys. 81, 163 (2009).

[20] Y. Huismans, A. Rouzée, A. Gijsbertsen, J. H. Jungmann, A. S. Smolkowska, P. S. W. M. Logman, F. Lépine, C. Cauchy, S. Zamith, T. Marchenko et al., Science 331, 61 (2011).

[21] W. Boutu, S. Haessler, H. Merdji, P. Breger, G. Waters, M. Stankiewicz, L. J. Frasinski, R. Taieb, J. Caillat, A. Maquet et al., Nat. Phys. 4, 545 (2008).

[22] D. Shafir, Y. Mairesse, D. M. Villeneuve, P. B. Corkum, and N. Dudovich, Nat. Phys. 5, 412 (2009).

[23] G. G. Paulus, W. Becker, W. Nicklich, and H. Walther, J. Phys. B 27, L703 (1994).
[24] P. Colosimo, G. Doumy, C. I. Blaga, J. Wheeler, C. Hauri, F. Catoire, J. Tate, R. Chirla, A. M. March, G. G. Paulus et al., Nat. Phys. 4, 386 (2008).

[25] X.-B. Bian and A. D. Bandrauk, Phys. Rev. Lett. 108, 263003 (2012).

[26] T. Zuo and A.D. Bandrauk, Phys. Rev. A 52, R2511 (1995).

[27] T. Zuo, A. D. Bandrauk, and P. B. Corkum, Chem. Phys. Lett. 259, 313 (1996).

[28] A. D. Bandrauk and S. Chelkowski, J. Mol. Struct. 591, 199 (2002).

[29] M. Meckel, D. Comtois, D. Zeidler, A. Staudte, D. Pavičić, H.C. Bandulet, H. Pépin, J.-C.C. Kieffer, R. Dörner, D. M. Villeneuve et al., Science 320, 1478 (2008).

[30] S. Baker, J.S. Robinson, C. A. Haworth, H. Teng, R. A. Smith, C. Chirilǎ, M. Lein, J.W. G. Tisch, and J.P. Marangos, Science 312, 424 (2006).

[31] M. F. Kling, C. Siedschlag, A. J. Verhoef, J. I. Khan, M. Schultze, T. Uphues, Y. Ni, M. Uiberacker, M. Drescher, F. Krausz et al., Science 312, 246 (2006).

[32] G. Sansone, F. Kelkensberg, J. F. Pérez-Torres, F. Morales, M. F. Kling, W. Siu, O. Ghafur, P. Johnsson, M. Swoboda, E. Benedetti et al., Nature (London) 465, 763 (2010).

[33] I. Sánchez and F. Martín, Phys. Rev. Lett. 79, 1654 (1997).

[34] J. Ullrich, R. Moshammer, R. Dorner, R. Dörner, L. P. H. Schmidt, and H. Schmidt-Böcking, Rep. Prog. Phys. 66, 1463 (2003).

[35] A. González-Castrillo, A. Palacios, F. Catoire, H. Bachau, and F. Martín, J. Phys. Chem. A 116, 2704 (2012).

[36] R. E. F. Silva, P. Rivière, and F. Martín, Phys. Rev. A 85, 063414 (2012).

[37] P. Rivière, R. E. F. Silva, and F. Martín, J. Phys. Chem. A 116, 11304 (2012).

[38] C. B. Madsen, F. Anis, L. B. Madsen, and B. D. Esry, Phys. Rev. Lett. 109, 163003 (2012).

[39] F. Martín, J. Fernández, T. Havermeier, L. Foucar, T. Weber, K. Kreidi, M. Schöffler, L. Schmidt, T. Jahnke, O. Jagutzki et al., Science 315, 629 (2007).

[40] D. Dowek, J. F. Pérez-Torres, Y. J. Picard, P. Billaud, C. Elkharrat, J. C. Houver, J. L. Sanz-Vicario, and F. Martín, Phys. Rev. Lett. 104, 233003 (2010).

[41] M. Šindelka, N. Moiseyev, and L. S. Cederbaum, J. Phys. B 44, 045603 (2011).

[42] K. Nagaya, Y. Teranishi, and H. Nakamura, J. Chem. Phys. 113, 6197 (2000).

[43] K. J. Schafer and K. C. Kulander, Phys. Rev. 42, 5794 (1990).

[44] F. Catoire and H. Bachau, Phys. Rev. A 85, 023422 (2012).

[45] B. Feuerstein and U. Thumm, Phys. Rev. A 67, 043405 (2003).

[46] A. Palacios, H. Bachau, and F. Martín, Phys. Rev. A 74, 031402(R) (2006).

[47] To keep the analogy with the full-dimensional $\mathrm{H}_{2}^{+}$ molecule, we use the notation $1 s \sigma_{g}$ and $2 p \sigma_{u}$ for the lowest electronic states, although in the lower dimensionality used in this work, only the inversion symmetry is meaningful. 